\title{
Nordic Walking Using Foot Weights to Increase Athletic Performance in Running
}

\author{
Nagovitsyn R.S. \\ Department of Physical Culture and Life Safety \\ Glazov State Pedagogical Institute \\ Glazov, Russia \\ gto18@mail.ru
}

\author{
Panachev V.D. \\ Department of Physical Culture \\ Perm National Research Polytechnic University, \\ Perm, Russia \\ panachev@pstu.ru
}

\author{
Osipov A. Yu. \\ Department of Physical Culture \\ Siberian Federal University, \\ V.F. Voyno-Yasenetsky State Medical University, Krasnoyarsk \\ Siberian Law Institute of the Ministry of Internal Affair of Russia, \\ Krasnoyarsk, Russia \\ ale44132272@yandex.ru
}

\begin{abstract}
Aim of the study: to analyze the effect of Nordic walking lessons of the 50-60 years elderly age using weights for the legs to increase the sporting result in running 2000 meters according to the standards of the ninth step of the "Ready for Labor and Defense" complex. Comparative analysis of people aged 50-60 years $(n=28)$, body weight 61-73 kilograms, regardless of gender, regularly engaged in Nordic walking: the experimental group $(n=14)$ used special weighting materials for the study legs during the passage of the distance, the control group $(n=14)$ was engaged in Nordic walking without the use of weighting agents during the experiment. Mathematical-statistical data processing was performed using Chi-square (X2) at $\mathbf{p}<0.05$. The use of a specially-developed training system of a 25 -week cycle with weights weight differentiation on each leg, the time taken to complete a distance and pace of movement, controlled by a heart rate monitor, revealed positive dynamics. The implementation of the author's training process with the use of weighting for the legs is more effective in the run for 2000 meters. This is reliably $(\mathbf{p}<0.05)$ was confirmed by comparing the numerical indicators of study participants after the experiment. In the experimental group, as compared with the control group, the number of athletes who fulfilled the standard in the 2000 meter race for the golden sign "Ready for Labor and Defense" increased significantly. In turn, in the control group, the number of athletes who completed the standard in the 2000-meter race for the bronze mark in comparison with the experimental group has increased significantly. The exercise organization offered in the study motivates and engages people of old age in competitive physical activity, taking into account their individual aerobic characteristics and emotional comfort. The proposed provisions and conclusions create prerequisites for further study of the individually differentiated physical education realization phenomenon and sports activities with elderly people in methodological, substantive and organizational aspects. The presented practical experience in the implementation of Nordic walking with the use of weighting for the legs can be used in the training process to prepare for the competition to identify the level of endurance involved in different ages.
\end{abstract}

Keywords-nordic walking; aged 50-60 years; weighting for the legs; endurance; "Ready for Labor and Defense".

\section{INTRODUCTION}

A distinctive feature of today's human life with everincreasing rhythms and a constant lack of time put this kind of human movement, like walking, into the background [1]. A person today is required to travel by public or personal transport, as well as other devices for movement [2]. People began to forget what it was like to go out and walk from home to work or just go for a walk [3]. And if walking is used in a person's life, it is often in uncomfortable shoes and clothes, very often with a burden and burden, disrupting the correctness of movements, provoking various diseases [4]

Many theoretical and methodical studies prove that it is necessary to engage in systematic and varied physical exercises, in particular, a simple form of fitness, like walking $[3,5,6]$. Walking is one of the most natural, familiar ways of human movement [7]. This is an automated motor act, carried out as a result of the trunk and limbs skeletal muscles coordinated activity complex [8]. You can walk regularly from any length of time, at any pace, any time of day and any day of the week. And as long as it lasts as comfortable or as free time [9] allows.

Walking is a good form of fitness that does not require large financial investments and a time frame. The main thing is to plan the lesson, think it over [8]. And it can become a passing physical activity during the main human activity [3] When walking, almost all muscle groups are involved, especially the muscles of the legs, back and abdomen. When compared with running, the musculoskeletal system, when walking, works sparingly. It has a positive effect on the cartilage tissue of the leg joints [10]. And this is most relevant for older people and having excess mass [2]. One of the most 
important loads on the human body when practicing walking falls on the cardiovascular system. Walking is essentially a kind of pacemaker [1].

In turn, as one of the most popular sports types of walking, is the Scandinavian or Nordic walking [5]. Nordic walking is a type of fitness that covers all age groups and muscle groups. Not only the muscles of the legs, back and abdomen, but the muscles of the arms and shoulder girdle, which during normal walking only have an indirect load [11]. It can be practiced at any age and in any physical form as a disease prevention, to improve individual physical fitness and as a basis for the formation of a healthy lifestyle [12].

Nordic walking activates at least $90 \%$ of all muscles in the body, while about $45 \%$ of muscles is used for normal walking. Nordic walking perfectly develops the shoulder girdle, the muscles of the back, arms and legs [10]. Depending on the walking speed, it burns about $46 \%$ more calories than with normal walking [9]. This is achieved by including the upper body in the training process.

The effectiveness of such a workout is extremely high: the work of the cardiovascular and respiratory systems improves, blood pressure returns to normal, the muscles of the whole body develop well, and endurance is improved [1]. In addition, Nordic walking reduces the load on the joints of the legs, it is also perfect for correcting posture. It is also believed that Nordic walking is an excellent exercise for weight loss and allows you to form a slender figure [10].

When implementing Nordic walking, not only special sticks (nordics), comfortable shoes and clothes suitable for the given season are used, but also various technologies that enhance the health and fitness effect [13]. Such, for example, as special training equipment, non-traditional sports equipment and conditions for the passage of the distance (special treadmills, weights, river sand, snow, climbing the mountain, etc.).

However, long-term aerobic exercise requires a long time due to low energy consumption, walking in this aspect has a certain temporary disadvantage [14]. To counter the lack of this exercise, which takes a long time, ways to increase exercise consumption during the same period of time is introduced. It can save time and increase the intensity and effectiveness of the exercise [15]. Examples include walking with dumbbells in your hands or carrying sandbags around each ankle [16]. In particular, many people perform heavy exercises with the use of sandbags, because sandbags are inexpensive and easy to carry. Walking with additional weight is considered more efficient than walking [17].

A preliminary analysis of research on the stated research problem revealed that in the current situation of popularizing Nordic walking among the population, a key contradiction is revealed $[5,18]$. Between a wide range of scientific research to improve the physical culture and healthy lifestyles of the population by means of Scandinavian walking and insufficiently developed methodological foundations for the implementation of this type of walking to improve athletic performance in endurance running based on the introduction of special weighting. In particular, improving the performance of middle-aged people (50-60 years) in the $2000 \mathrm{~m}$ distance race according to the standards of the ninth stage of the AllRussian sports complex "Ready for Labor and Defense".

Thus, the aim of the study: to analyze the impact of Nordic walking lessons of the 50-60 years elderly age using weighting for the legs to increase the sports result in running 2000 meters according to the standards of the IX stage of "Ready for Labor and Defense".

\section{MATERIALS AND METHODS}

\section{A. Participants:}

People of the 50-60 years elderly age $(n=28)$, body weight 61-73 kilograms, regardless of gender, regularly engaged in Nordic walking. Which at least four times a week are actively engaged in physical exercise and have no contraindications to the maximum physical exertion of an aerobic nature. Participants of the study were divided into two groups according to their personal wishes. The experimental group $(n=14)$ used during the experimental work special weighting for the legs during the passage of the distance. In turn, the control group $(n=14)$ was engaged in Nordic walking without using weighting for a certain period of time for the experiment.

\section{B. Organization of the research}

The experiment was implemented on the basis of the Progress sports complex and the Children's and Youth Sports School No. 1 in the city of Glazov (Udmurt Republic, Russia). To determine the level of physical development, the standards of the All-Russian sports and fitness complex "Ready for Labor on Defense" (RLD) were used according to the 9th step standards (bronze, silver and gold badges): running 2000 meters and Nordic walking 3000 meters. Before the experiment (April 2018) and after the experiment (October 2018), preliminary and control testing of all subjects was carried out, respectively. For 6 months, all participants in the experiment implemented the training process in Scandinavian walking at a special stadium, forest parks, city and country parks and squares.

In the experimental group, the participants in the study at one workout per week in the main part of the Nordic walking class used special weighting pads for the legs $(0.25-2 \mathrm{~kg})$ during the passage of the distance. Weighting for the legs this is a special cuff with sewn weight, which are put on the ankles and give additional stress when performing physical exercises. For the experiment were used two types of weighting. Bulk weights are a small bag of fabric that is filled with sand, metal chips or other crumbly material. In the plate weighting for the legs iron plates were used as a load. They were inserted into special pockets that are sewn onto a thick canvas. The weight of the plate weights was regulated with the help of additional metal plates, which was very convenient for the implementation of occupations.

The research participants in the experimental group changed the weight of weighting on each leg, the time of the distance and the pace of movement passage, controlled by the 
the possibility of injury to an athlete without the necessary training.

Statistical analysis: mathematically-statistical data processing was performed using chi-square (X2) with $\mathrm{p}<0.01$ and $p<0.05$ taking into account the normality of the sample distribution. Before the start of the experiment, the mathematical-statistical uncertainty of the differences between the experimental (EG) and control (CG) groups with p> 0.05 at distances of 2000 meters and Nordic walking of 3000 meters was recorded.

\section{RESULTS}

The analysis of the data obtained by the subjects was ranked according to the standards of tests (tests) of the AllRussian physical training and sports complex "Ready for Labor on Defense" according to the ninth stage. As a result,

the study participants were divided into three level groups depending on the correspondence of their result in bronze, silver or gold insignia. The results of control testing at distances of 2000 meters running and Nordic walking of 3000 meters are presented in Figure 1-2:

- calculation of the maximum pulse value by the formulas for people of the elderly: men 214 - age * 0.8 and women 209 - age * 0.7

- determine the different rates of passing the distance in three levels. Aerobic level - 70-80\% of the maximum value of the pulse. Developmental load in this area allows you to increase the body's capabilities, using the energy from the breakdown of adenosine-triphosphate with oxygen. Anaerobic level $-80-90 \%$ of the maximum pulse value. This is the most difficult "oxygen-free" training zone. Used by systematically practicing athletes to train special endurance. It is accompanied by the splitting of adenosine-triphosphate without oxygen and the release of lactic acid. Extreme level $90-100 \%$ of the maximum value of the pulse. It allows you to develop explosive force, it is performed with maximum intensity and short duration from several seconds to 2 minutes. A feature of training in this area is that the body spends its resources to the maximum. Accompanied by high fatigue and

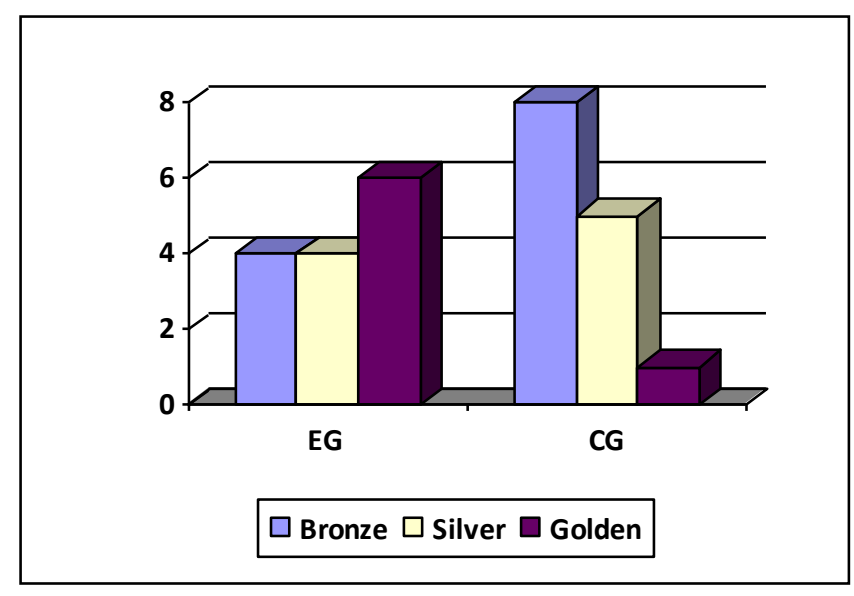

$\square$ Bronze $\square$ Silver $\square$ Golden

Fig. 1. Test results in the 2000 meter race on the control test

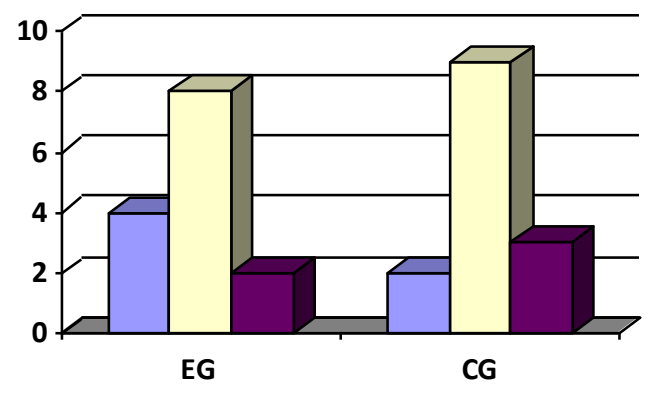

$\square$ Bronze $\square$ Silver $\square$ Golden

Fig. 2. Test results Nordic walking at 3000 meters on the control test 
The results of testing participants in the 2000 meter race showed that the implementation of the author's training process using weighting for the legs is more effective. What is reliable $(\mathrm{p}<0.05)$ was confirmed by comparing the numerical indicators of the study participants after the experiment. Moreover, prior to the experiment, a non-significant $(\mathrm{p}>0.05)$ comparative result was obtained between the experimental and control groups. In the experimental group, as compared with the control group, the number of athletes who fulfilled the standard in the 2000 meter race for the golden sign "Ready for Labor and Defense" increased significantly. In turn, in the control group, the number of athletes who completed the standard in the 2000-meter race for the bronze mark compared with the experimental group, is significantly larger. In general, the mathematical-statistical analysis of the participants number in three level groups revealed that the effectiveness of the experimental group of respondents during the experiment significantly increased $(\mathrm{p}<0.05)$, and in the control group remained statistically unchanged $(p>0.05)$ for the test in the 2000 meters race.

Analysis of the participants testing in the study in the Scandinavian walking at 3000 meters showed that the implementation of the author's training process using weights for the feet does not affect the performance in the implementation of this standard. Not significant results were recorded $(p>0.05)$ when comparing the numerical indicators of the study participants before and after the experiment between the experimental and control groups. In the experimental group, compared with the control group, the number of athletes who fulfilled the standard in the Scandinavian walk of 3000 meters on the gold sign "Ready for Labor and Defense" did not significantly increase. In turn, in the control group, the number of athletes who performed the standard in the Scandinavian walk of 3000 meters on a bronze sign compared with the experimental group, is not significantly higher. In general, the mathematical-statistical analysis of the participants number in the three level groups revealed that the effectiveness of the experimental and control group of respondents during the experiment did not increase $(p>0.05)$.

\section{DISCUSSIONS}

In general, the results of the study are consistent with the results of other studies on the impact of active Nordic walking on the effectiveness of the implementation of the training process for people of various ages and sports categories $[3,5$, 10]. The researchers experimentally substantiate the improvement of physical fitness indicators young people and people of middle and old age and health state [2,19], carried out using the innovative extended motor mode in Nordic walking [12]. As shown by experimental studies [20], the effect of Scandinavian walking compared to traditional walking exercises and exercises on resistance of the group on physical fitness in the elderly is more reliable. Classes for Nordic walking have a positive effect on motivation for physical activity through the improvement of anthropometric parameters, body composition, cardiovascular processes and blood composition [9].

Recently, many authors have statistically proved that Nordic walking, focused on the physical development of students, affect cognitive thinking operations [11]. That the systemic activities of Nordic walking interfere with depressive states and motivate a person to lively activity [11]. Certain aspects in the author's study of an individually differentiated program update the physical culture and sports reference points of the recreational activity of middle and pre-elderly people $[3,21]$. In particular, research work is done on the comparative analysis of sports running and various types of walking in the middle and maximum modes of passing the distance [18]. Therefore, this study does not contradict these trends and proposes an original approach to training for those engaged in running for medium or long distances through the training process in walking, particularly in Nordic walking, based on the introduction of a special additional load.

The authors of the article agree with the opinion of a number of foreign experts on the impact of individualization and differentiation in the training process on Nordic walking to improve various physical qualities of athletes of different ages [22], in particular, the flexibility of the upper and lower body parts, upper body strength, cardiovascular endurance [20]. The results obtained in the study, on the one hand, reveal the features of the special weighting agents introduction in the implementation of aerobic exercise. Through a conscious understanding of its application content, as well as ownership of the forms and methods of physical culture with it. There are empirical studies on the positive introduction of additional stress on the ankle of the affected side as a clinical intervention to improve the balance of patients with hemiplegic stroke [23]. Scientists have experimentally proved that various levels of aggravating stress can be effective in enhancing the factors of walking in adults without diseases [8]. On the other hand, to a systemic understanding of the physical culture improvement and sports activities to develop such a necessary quality as endurance through weights. Studies show that special training conducted at different levels of motor activity reliably have a significant impact on performance $[24,25]$. Which include walking with dumbbells in your hands or wearing sandbags around each ankle. An experiment was conducted regarding the weight load on the lower limbs. The study showed that physical effects are enhanced when the place of the load attachment to weight is closer to the lower limb than to the upper limb, and that the minimum load on the weight is $2 \%$ of body weight [16].

However, as the study [12] shows, the excessive use of additional equipment during the implementation of Nordic walking, especially those who are not trained, can lead to various types of injuries. In this aspect, the effect of weighting agents of different weight categories on various functional indicators of different age and sex people will be the vector of our further research.

\section{CONCLUSIONS}

Thus, the use of a specially-developed system of a 25 week training cycle with weights weight differentiation on each leg, the time taken to go the distance and the pace of movement, controlled by the heart rate monitor, revealed a positive trend. The study statistically proved that Nordic walking lessons of the elderly age of 50-60 years using weights for the legs increase the athletic performance in the 
[8] J.W. Hwang, S.K. Lee, J.S. Park, S.H. Ahn, K.J. Lee and S.J. Lee, The effects of ankle weight loading on the walking factors of adults without symptoms. J Exerc Rehabil, vol. 13 (4), 2017, pp. 425-429, doi: 10.12965/jer.1734954.477. of the "Ready for Labor and Defense" complex. In the experimental group, as compared with the control group, the number of athletes who fulfilled the standard in the 2000 meter race for the golden sign "Ready for Labor and Defense" increased significantly.

Our study is consistent with other scientific studies of this subject. The proposed organization of physical exercise classes in the study motivates and involves people of old age in competitive physical culture and sports activities, taking into account their individual aerobic characteristics and emotional comfort. Takes into account their level of physical fitness and physical development as an interconnection of skills, allowing them to positively realize physical culture activities. However, no matter what kind of walking a person is carried away with, no matter what physical exertion he feels, Nordic walking should bring visible results, both in the form of spiritual satisfaction and sincere physical joy, and in the development of his physical abilities and physical fitness. To achieve all the goals, it is first of all necessary to deal with positive motivation and systematically.

The proposed provisions and conclusions create prerequisites for further study of the individually differentiated physical education and sports activities realization phenomenon with elderly people in methodological, substantive and organizational aspects. The presented practical experience in the implementation of Nordic walking with the use of weighting for the legs can be used in the training process to prepare for the competition to identify the level of endurance involved in different ages.

\section{References}

[1] Y.A. Kopylov, The Scandinavian walking with sticks. Physical education at school, vol. 6, 2014, pp. 47-50.

[2] H. Figard-Fabre, N. Fabre, A. Leonardi, A. Leonardi and F. Schena, Efficacy of Nordic Walking in Obesity Management. International journal of sports medicine, vol. 32, iss. 6, 2011, pp. 407-414. doi: 10.1055/s-0030-1268461.

[3] M. Tschentscher, D. Niederseer and J. Niebauer, Health Benefits of Nordic Walking A Systematic Review. American journal of preventive medicine, vol. 44 (1), 2013, pp. 76-84, doi: 10.1016/j.amepre.2012.09.043

[4] P.B. Volkov and R.S. Nagovitsyn, Gaming technologies in the development of spine flexibility and the coordination of children and teenagers' movements in sports classes in country health camps. Pedagogics psychology medical-biological problems of physical training and sports, vol. 22, iss. 1, 2018, pp. 50-55, doi: 10.15561/18189172.2018.0107.

[5] V. Bullo, S. Gobbo, B. Vendramin, F. Duregon, L. Cugusi, A. Di Blasio, D.S. Bocalini, M. Zaccaria, M. Bergamin and A. Ermolao, Nordic Walking Can Be Incorporated in the Exercise Prescription to Increase Aerobic Capacity, Strength, and Quality of Life for Elderly. A Systematic Review and Meta-Analysis. Rejuvenation Research, vol. 21 , iss. 2, 2018, pp. 141-161, doi: 10.1089/rej.2017.1921

[6] R.S. Nagovitsyn, P.B. Volkov and A.A. Miroshnichenko, Planning of physical load of annual cycle of students', practicing cyclic kinds of sports, training. Physical education of students, vol. 21, iss. 3, pp.126133, doi: 10.15561/20755279.2017.0305

[7] S.N. Whittlesey and J. Hamill, An alternative model of the lower extremity during locomotiom. J Appl Biomech, vol. 12, 1996, pp. 269279.

9] S. Gobbo, V. Bullo, E. Roma, F. Duregon, D.S. Bocalini, R.L. Rica, A. Di Blasio, L. Cugusi, B. Vendramin, M. Bergamo, D. Cruz-Diaz, C.L. Alberton, A. Ermolao and M. Bergamin, Nordic Walking promoted weight loss in overweight and obese people: A systematic review for future exercise prescription. Journal of Functional Morphology and Kinesiology, vol. 4 (2), 2019, pp 36-37, doi: $10.3390 /$ jfmk4020036.

[10] J.M. Shim, H.Y. Kwon, H.R. Kim, B.I. Kim and J.H. Jung, Comparison of the effects of walking with and without Nordic pole on upper extremity and lower extremity muscle activation. Journal of Physical Therapy Science, vol. 25, iss. 12, 2013, pp. 1553-1556, doi https://doi.org/10.1589/jpts.25.1553.

[11] K. Sujika, U. Pechter, R. Kaida, H. Tähepõld, J. Maaroos and H.I. Maaroos, Physical Activity of depressed patients and their motivation to exercise: Nordic walking in family practice. International Journal of Rehabilitation Research, vol. 32, 2009, pp. 132-138.

[12] K. Knobloch and P.M. Vogt, Nordic pole walking injuries - Nordic walking thumb as novel injury entity. Sportverletzung-sportschaden, vol. 20, iss. 3, 2006, pp. 137-142, doi: 10.1055/s-2006-926995.

[13] I.V. Bogdanov, Start walking! Physical education at school, vol. 3, 2015 pp. 61-63

[14] Y.K. Kim, Y.K. Kwon and Y.W. Jang, Physical response in treadmill walking exercise after artificial weight loading. Korean J Sport Med, vol. 20, 2002, pp. 72-81.

[15] T.E. Auble and L. Schwartz, Physiological effects of exercising with handweights. Sports Med, vol. 11(4), 1991, pp. 244-56.

[16] J.S. Yang, Physiological responses to walking exercise with added weight at different locations in normal subjects. Korean J Phys Educ, vol. 35, 1996, pp. 130-143.

[17] R.L. Burse, K.B. Pandolf and R.F. Goldman, Physical conditioning of sedentary young men with ankle weights during working hours. Ergonomics, vol. 22 (1), 1979, pp. 69-78.

[18] K. Sasaki and R.R. Neptune, Differences in muscle function during walking and running at the same speed. Journal of Biomechanics, vol 39, iss. 11, 2006, pp. 2005-2013, doi: 10.1016/j.jbiomech.2005.06.019.

[19] G. Kim and N.M Yoon, A study on kinetic gait analysis of the normal adult. J Korean Soc Phys Ther, vol. 21, 2009, pp. 87-95.

[20] N. Takeshima, M.M. Islam, M.E. Rogers, N.L. Rogers, N. Sengoku, D Koizumi, Y. Kitabayashi, A. Imai and A. Naruse Effects of Nordic Walking compared to Conventional Walking and Band-Based Resistance Exercise on Fitness in Older Adults. Journal of sports science and medicine, vol. 12, iss. 3, pp. 422-430.

[21] R.S. Nagovitsyn, A.A. Miroshnichenko, D.R. Merzlyakova and G.Z Faizullina, Interrelation of mental "burn out" level and psychological health in athletes with different qualification. Physical education of students, vol. 22, iss. 6, pp. 327-331. doi: 10.15561/20755279.2018.0608.

[22] D.J. Kwak, K.T. Kim, G.M. Kang, , Y.J. Park, H.L. Lee and M.K. Kim, Effect of 8-week Nordic walking training on nondominant hand grip and shoulder strength in middle-aged women. Journal of exercise rehabilitation, vol. 15, iss. 3,2019 , pp. 414-418, doi: 10.12965/jer.1938226.113.

[23] J.H. Park, G. Hwangbo and J.S. Kim, The effect of treadmill-based incremental leg weight loading training on the balance of stroke patients. Journal of Physical Therapy Science, vol. 26, iss. 2, 2014, pp. 235-237, doi: $10.1589 /$ jpts.26.235.

[24] A. Virág, C.K. Karóczi, A. Jakab, Z. Vass, E. Kovács and T. Gondos, Short-term and long-term effects of nordic walking training on balance, functional mobility, muscle strength and aerobic endurance among Hungarian community-living older people: a feasibility study. J Sports Med Phys Fitness, vol. 55, 2015, pp. 1285-1292.

[25] S.K. Park, D.J. Yang, Y.H. Kang, J.H. Kim, Y.H. Uhm and Y.S. Lee, Effects of Nordic walking and walking on spatiotemporal gait parameters and ground reaction force. J Phys Ther Sci, vol. 27 (9), 2015 2891-2893, doi:10.1589/jpts.27.2891. 


\section{PRESS}

[26] Mohamed Bennadja, Kheiredine Benrabah, Kharoubi Mohamed Fayçal, Ouadah Ahmed el Amine. The effects of pylometric training on sprint performance and Repeated Sprint Ability of Futsal players. The Russian
Journal of Physical Education and Sport. 2018, 13(1), pp. 20-28. DOI: 10/14526/01_2018_280. 